\title{
Congenital Unilateral Infiltrating Facial Lipomatosis
}

The Editor,

Sir,

Slavin et al in 1983 described Congenital diffuse infiltrating lipomatosis of the face (CDIL-F), which is an unfamiliar clinical entity characterized by collections of nonencapsulated, mature adipocytes that infiltrate locally, leading to craniofacial deformities with a tendency for recurrence after surgery (1). Affected children do not have abnormal psychomotor development but the facial asymmetry causes cosmetic concerns. We present the radiological findings in our case.

A one-year-old female presented with progressively increasing swelling over the right face since birth with the early eruption of deciduous teeth. The swelling on the right side of the face was soft, non-tender, ill-defined and diffuse causing unilateral facial hypertrophy (Fig. 1).

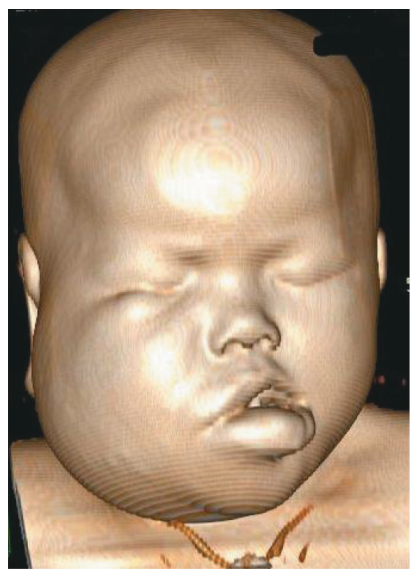

Fig. 1: Computed tomographic soft-tissue rendered three-dimensional volumetric reconstruction. There is right facial asymmetry and large preauricular and cheek swelling.

Computed tomography of the head and face showed fatty infiltration (-25 to -100 Hounsfield units) of the right half of the cheek, upper and lower lips, buccinators, masseters, parapharyngeal space and infratemporal fossa (Fig. 2).

The right parotid gland showed fatty atrophy with lipomatous infiltration of the right half of the tongue. The right hemi-mandible was enlarged, distorted and showed fatty attenuation within it. Magnetic resonance imaging (MRI) confirmed the fatty infiltration and showed the absence of areas of soft-tissue intensity (Fig.3). Biopsy through the right cheek showed unencapsulated, lobulated, infiltration of mature fat cells without any atypical cells. Based on the clinical, radiological and histopathological features, a diagnosis of congenital diffuse infiltrating lipomatosis of the face (CDIL-F) was made.

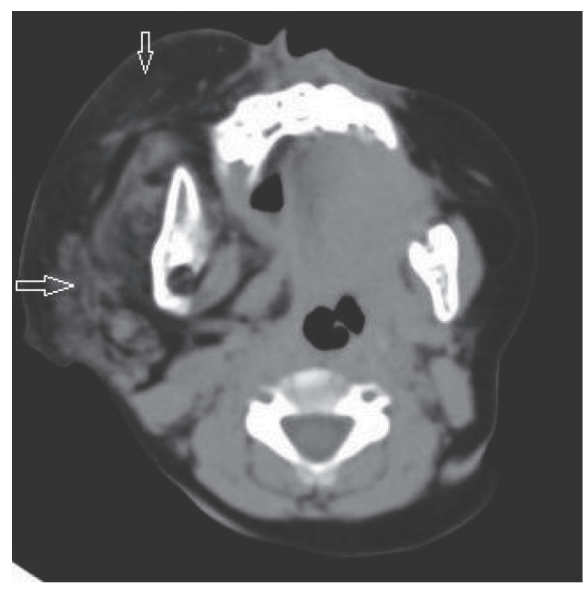

Fig. 2: Axial non-contrast computed tomography. There is fatty infiltration in the right buccinator region (down arrow) with lipomatous atrophy of parotid gland (right arrow).

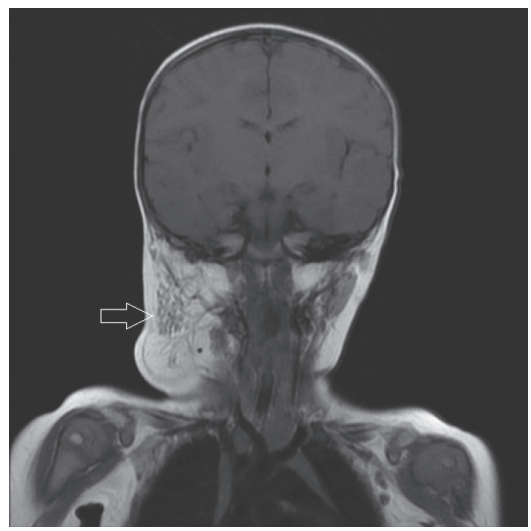

Fig. 3: Coronal T1 Weighted MRI. There are hyperplastic subcutaneous fat and diffuse fatty infiltration in the right buccal, masseteric, parotid and parapharyngeal spaces.

Congenital diffuse infiltrating lipomatosis of the face is characterized by nonencapsulated proliferation of mature adipose tissue causing diffuse infiltration of muscle and adjacent soft-tissue. There are presence of fibrous tissue, nerve bundles and vessels with a thickened wall without lipoblasts and signs of malignancy. Skeletal findings in CDIL-F are sclerosis and the hyperplasia of the skull, cervical vertebrae, hemimandibular hyperplasia (of the ramus and/or condyle), accelerated dentoskeletal growth and zygomatic hyperplasia (2).

Differential diagnoses of CDIL-F are lymphatic or vascular malformations, lipomas (intermuscular/intramuscular, angiolipomas/fibrolipomas, liposarcoma and lipoblastomatosis) and congenital overgrowth syndromes causing progressive hemifacial hyperplasia. 
The role of sonography, computed tomography (CT) and magnetic resonance imaging (MRI) is very contributory in making the diagnosis. Ultrasound helps in identifying the diffuse, fatty, infiltrative and non-vascular nature of the lesion but its true anatomical extent can be depicted by cross-sectional imaging only. Computed tomography scan shows a diffuse, non-uniform fatty infiltration with Hounsfield Unit being in the negative range of fat along with a unilateral hyperplasia of craniofacial bones as seen in the present case. The predominantly fatty nature of the condition is confirmed on MRI. In children, the lesion exhibiting high signal intensity (isointense to fat) on $\mathrm{T} 1$ and $\mathrm{T} 2$-weighted images and hypointensity on fat-suppressed sequences along with strong clinical correlation eliminate the need for biopsy.

This entity is managed primarily by wide local excision
Keywords: Congenital, face, lipomatosis

SV Kashikar

From: Department of Radiology, Jawaharlal Nehru

Medical College, Sawangi Meghe, Wardha, India.

Correspondence: Dr SV Kashikar, Behind Magnanwadi, Ramnagar, Wardha, Maharashtra 442001, India. Email: shivalikashikar@gmail.com

\section{REFERENCES}

1. Slavin SA, Baker DC, McCarthy JG, Muffarij A. Congenital infiltrating lipomatosis of the face: clinicopathologic evaluation and treatment. Plast Reconstr Surg1983; 72: 158-64.

2. Bouletreau P, Breton P, Freidel M. Congenital infiltrating lipomatosis of the face: case report. J Oral Maxillofac Surg 2000; 58: 807-10.

3. Padwa BL, Mulliken JB. Facial infiltrating lipomatosis. Plast Reconstr Surg $2001 ; 108$ : $1544-4$. 